\title{
Measuring the vulnerability of public transport networks
}

\author{
Eduardo Rodríguez-Núñez, Juan Carlos García-Palomares
}

\begin{abstract}
In the last years, studies on the vulnerability of public transport networks attract a growing attention because of the repercussions that incidents can have on the day-to-day functioning of a city. The aim of this paper is to develop a methodology for measuring public transport network vulnerability taking the Madrid Metro system as an example. The consequences of a disruptions of riding times or the number of missed trips are analysed for each of the network links with a full scan approach implemented in GIS (Geographic Information Systems). Using real trips distribution, each link in the network is measured for criticality, from which the vulnerability of lines and stations can be calculated. The proposed methodology also makes it possible to analyse the role of circular lines in network vulnerability and to obtain a worst-case scenario for the successive disruption of links by simulating a targeted attack on the network. Results show the presence of critical links in the southern part of the network, where line density is low and ridership high. They also highlight the importance of the circular line as an element of network robustness.
\end{abstract}

\section{Introduction}

Public transport networks are essential for mobility in urban areas. According to the International Association of Public Transport (UITP), in 2008 around 60 billion trips were made on public transport in the cities of the 27 member states of the European Union. In order to guarantee the efficient running of these networks, transport planners need to know their level of vulnerability to possible incidents and identify those parts of the networks where incidents will have the greatest impact on passengers.

Any type of incident occurring on a public transport network will affect the day-to-day functioning of the city itself. The effects are particularly felt when rail travel is involved because of the amount of passengers who use this means of transport and its greater vulnerability. Train breakdowns, electrical failures and engineering work may lead to temporary line closure, either in one or both directions, and incidents such as suicides, demonstrations or strikes may also affect the frequency of the service. More dramatic events like terrorist attacks, such as those that took place in London and Madrid, have particularly far-reaching and longlasting effects on the network. The terrorist attack on London's subway and bus system produced a tremendous disruption in the lives of London residents. Several months after the subway attack, weekend ridership was still down by $30 \%$ and between $5 \%$ to $15 \%$ on weekdays (Chen et al., 2007).

It is in this context that network vulnerability studies are becoming increasingly important. Vulnerability is related to the capacity to continue operating following disruption, in other words, the degree of susceptibility of a network to certain incidents that may lead to reduced service or accessibility levels (Berdica, 2002). These incidents will have a greater impact on some links of the networks than on others. A network node is vulnerable if loss (or substantial degradation) of a small number of links significantly diminishes the accessibility of the node, as measured by a standard index of accessibility (Taylor et al., 2006).

It is therefore necessary to know the criticality as well as the vulnerability. The critical elements of a network (links or nodes) are those that most affect its vulnerability: the more critical the element, the greater the effects of its loss on the system (Taylor et al., 2006; Jenelius et al., 2006). The criticality level of an element depends on the role it plays in the network structure and the flows within it. It is essential to be aware of the weak points and alternative routes in the network in order to mitigate vulnerability (Chen et al., 2007) or, if need be, give priority to some links over others for rebuilding after a catastrophic event (Sohn, 2006; Bono and Gutiérrez, 2011).

The metropolitan area of Madrid is a good example of the importance of public transport networks for mobility. Of the 
different modes of public transport in Madrid, the Metro has the greatest ridership. In 2010 it carried 630 million passengers out of a total of 1488 million who used public transport. Other modes of transport followed: urban buses (469 million passengers), interurban buses ( 189 million), the local train service ( 181 million) and the light railway (17 million).

The aim of this study is to measure criticality and vulnerability in a public transport network. To do this, the consequences of disruption in each link of the network are analysed in order to determine their impact on riding times or the unsatisfied travel demand. Unlike most studies on the vulnerability of public transport networks, which work with graph theory indicators, this study analyses increases in riding times caused by having to resort to alternatives to the optimum route, using actual network flow distribution. The worst scenarios for a successive disruption of links are also obtained by simulating a coordinated attack on the network, such as a possible terrorist attack or sabotage during a strike, and the repercussions on flow distributions deriving from such scenarios are shown. The case study used is the subway network in the city of Madrid (Madrid Metro), and GIS has been applied.

After this introduction (Section 1) the paper is organized as follows. Section 2 reviews the literature on transport network vulnerability analyses in general and in public transport in particular. Section 3 presents the methodology applied to analyse the Madrid Metro system. The characteristics of the network and data used are described in Section 4 and the results of the analysis are shown in Section 5. Section 6 consists of a discussion of the results and the final conclusions.

\section{Background}

Recent literature reflects a growing interest in network vulnerability studies. Contributions to the study of the vulnerability are produced in many different disciplines and have been applied in numerous fields (see, for example: Myung and Kim, 2004; Matisziw et al., 2012; Alderson et al., 2013). Transport literature has also incorporated vulnerability aspect in its studies. Especially after the terrorist attacks on New York, Madrid and London and natural disasters, such as earthquakes or hurricanes like Katrina and Rita, that affected transport networks over the last few years. Some reviews on this work describe attempts being made to establish theoretical, conceptual and methodological bases for studying transport network vulnerability. For example, in her review of the literature on road network vulnerability, Berdica (2002) puts particular emphasis on defining the concepts to use, discussing previous studies and exploring the way forward for future lines of study. Jen (2005) published a book on the design of robust networks. Robustness is understood to be the capacity of the network to absorb the impact of disturbances in its links and nodes while maintaining operability in conditions similar to those found in a normal situation. Murray and Grubesic (ed., 2007) examine the vulnerability of networks from different perspectives (geographical, economic and social). Other recent reviews focus on concepts such as resilience, defined as the capacity of networks to recover from a possible incident (Reggiani, 2013).

By and large, studies on transport network vulnerability can be differentiated according to whether they deal with road networks (the most frequent) or public transport (less usual). Two main indicator types are used for analysis. In studies related to the physical sciences or mathematics, graph theory and complex network indicators are applied, while vulnerability is usually measured from accessibility or serviceability indicators in studies concerned with transport and territory. Apart from the type of network considered or the indicators used, network vulnerability studies can be differentiated according to the approach adopted. Murray et al. (2008) proposed a basic typology of network vulnerability approaches, namely: scenario specific, strategy specific, simulation, and mathematical modelling. Scenario-specific approaches evaluate the potential ramifications of a specific disruption scenario or small set of scenarios. Strategy-specific approaches address questions such as: how vulnerable is a network to a structured or coordinated loss of facilities? In simulation-based approaches, the goal is to evaluate a suitable number of scenarios to obtain an effective characterization of the range of possible impacts. Finally, mathematical modelling approaches seek to identify those scenarios with the potential to most affect network operation with respect to the loss or hardening of facilities.

\subsection{Transport network vulnerability assessment}

Although public transport networks are more sensitive when it comes to vulnerability, until a few years ago most research was carried out on road networks, particularly when done from a geographical perspective or for transport planning purposes (see, for example: Berdica, 2002; Berdica and Mattsson, 2007; Chen et al., 2012; Erath et al., 2008; Jenelius, 2009, 2010; Jenelius et al., 2006; Jenelius and Mattsson, 2012; Taylor et al., 2006; Taylor and Susilawati, 2012). In these cases, a frequently used indicator was accessibility. In the meantime, with respect to public transport networks, the majority of authors applied graph theory indicators within the framework of more recent complex network theory. These indicators were already used in early studies on network vulnerability to measure the increase in topological distance resulting from disruption in certain links (Garrison, 1960) and to identify the most critical links of a network (Ratliff et al., 1975). However, the development of ever more efficient computer applications has led to a substantial increase in this type of study. Several examples exist for subway networks. Criado et al. (2007), Derrible and Kennedy (2010) study the form and structure of different subway networks, analysing as many as 66 and 33 networks respectively. Angeloudis and Fisk (2006) measure robustness in the face of possible random attacks in the 20 most important subway systems in the world. These indices have also been applied to more recent studies in which subway networks are considered not as a closed system but as a sub-network interacting with others, in a complex public transport system (Zhang and Peeta, 2011; von Ferber et al., 2012). Among these studies is the analysis by Mouronte and Benito (2012), who study the Madrid Metro and urban bus network, applying some of the most commonly used graph indicators (shortest distance between nodes, betweenness, detection of clusters and robustness). Also in the last few years, there have been some attempts to approach this type of analysis from a more geographical perspective. Bono and Gutiérrez (2011) have integrated concepts and network theory indicators to measure vulnerability and GIS tools for visualising and analysing results.

Some recent studies have tried to incorporate complementary information into graph theory indicators (see, for example, Mishra et al., 2012 or Matisziw et al., 2012). However, in most of them network structure analysis still prevails and they lack a more comprehensive analysis on the operation of public transport systems that brings together data on trips distribution, network capacity and the cost of travel (Matisziw et al., 2009). Nevertheless, this type of information should be included in order to know the vulnerability of a transport network. The introduction of a double perspective into vulnerability analysis has been advocated: that of network supply and demand (Berdica and Mattsson, 2007; Chen et al., 2007; Jenelius, 2009; Jenelius et al., 2011; Reggiani, 2013). The quickest way of doing this is to use accessibility indicators to analyse the impacts of incidents in terms of market potential or generalised transport costs, or serviceability indicators like average trip 
times. Cats and Jenelius (2012) make one of a few contributions of vulnerability public transport networks in this regard.

\subsection{Approaches adopted}

Studies on vulnerability have used different approaches to measure accessibility and serviceability. Accessibility has frequently been used to assess specific scenarios, particularly for road networks. The effects of disruption on one or more elements of the network in either real or hypothetical situations are simulated. This type of study enables a detailed analysis of each of the proposed scenarios to be carried out to assess impacts that are both direct (operation of the network) and indirect (economic consequences). Suárez et al. (2005) used a four-step travel models to analyse the impacts of possible flooding of the transport system in the Boston metropolitan area. Sohn (2006) applied accessibility indicators to assess how the Maryland network would respond to flood risk. Chang (2003) studied how accessibility gradually improved after the Kobe earthquake as sections of the rail network were re-established. ${ }^{1}$ Berdica and Mattsson (2007) assessed the impact of the closure of different bridges in Stockholm. Taylor et al. (2006) analysed the effects of the elimination of various sections of the Australian road network in terms of generalised cost increases and the loss of potential accessibility. Knoop et al. (2008), Erath et al. (2008) simulated the impacts of closing sections of the road networks in Rotterdam and Switzerland, respectively. Fang et al. (2012) used data on journeys made by up to 12,000 taxis to analyse the vulnerability of a series of bridges in the city of Wuhan, China, working with a trip assignment model and incorporating spatiotemporal prisms. Other studies analyse the economic impacts of the closure of certain transport axes, especially in transport corridors that form a backbone for important commercial relations. Such an example is the study by Masiero and Maggi (2012), who assess the cost, both in direct terms (generalised transport cost) and indirect terms, of the two-week closure of the north-south St. Gotthard road corridor, one of the most crucial infrastructure in Europe.

Along with specific scenario assessment, a second approach seeks to identify the most critical elements in a network, or the most vulnerable in the worst scenarios. This is done by using a full scan approach to analyze the role played by each element in the network operation. Jenelius and Mattsson (2005) have carried out a systematic study of how disrupted links affect a network by analysing a separate scenario for every link in the network. In another study on the road network in northern Sweden, they calculate indices that measure the importance of each link in the network and for exposure to vulnerability for each municipality in the region (Jenelius et al., 2006). They use a transport model with which they assign the total number of vehicles to each link in the network and then calculate increments in the generalised trip cost when each of these links is closed. Matisziw et al. (2009) identify more critical scenarios using combinations of the loss of several links in the Ohio road network. Scott et al. (2006) propose a robustness index to identify critical links and assess network operation, which they test on three hypothetical road networks. This index is used to measure changes in trip times associated with the reordering of all the traffic when one of the links becomes unusable. On a regional scale, Taylor and Susilawati (2012) set out to identify the most critical locations in a situation of degradation on a road network in south east Australia, using a remoteness indicator (the opposite of accessibility) to assess all the localities in the region. Another element of network vulnerability is increased congestion. Watling and Balijepalli (2012) measure the vulnerabil-

\footnotetext{
${ }^{1}$ The optimization approach for network restoration during disaster recovery has been studied in other fields, for example, by Matisziw et al., 2010 or Nurre and Sharkey, 2010.
}

ity of each link of the network to the effects of growth in demand. Chen et al. (2012) use a transport model to highlight vulnerability in congestion scenarios, but in this case the effects of each link are analysed in what they call its "impact area", which makes the analytical process quicker.

A third approach in transport network vulnerability studies is the strategy-specific assessment, the simulation of targeted attacks. As yet, there has been little work along these lines and the few studies that have been done use methodologies applied to other types of network not specific to transport (mostly information technology). Such scenarios are based on a sequence of losses of network elements, for example, those caused by a terrorist attack. The question posed is: how vulnerable is a network to a structured or coordinated loss of facilities? An initial approach was to rank links in order of importance (criticality), based on a topological criterion, and then eliminate them one by one in the same order, analysing the effects at each stage (Albert et al., 2000). In more recent studies, either the importance of a link is recalculated at each stage, or links and nodes are simply eliminated at random (Albert et al., 2004; Holme et al., 2002). Optimisation techniques and games theory have also been used in transport network studies to identify the worst scenarios and formulate a response (see, for example, Bell, 2000; Bell et al., 2008; Criado et al., 2004; Lou and Zhang, 2011). Alderson et al. (2013) show how to evaluate the criticality of sets of components, how to assess the worst-case set of components that might be lost to a given number of simultaneous hostile attacks (or engineering failures, or losses to Mother Nature), and how to allocate limited defensive resources to minimize the maximum damage from a subsequent attack. Johansson et al. (2007) study the different consequences between random and structural removal of nodes or links in an electric distribution network.

In this paper, serviceability is used to measure the criticality of each link of a public transport network and, with this information, analyse vulnerability at station level. This study incorporates the double perspective of network and demand, using data from real travel patterns. The three types of approach commonly used in vulnerability studies are coordinated through a simple serviceability indicator (weighted average time). A full network scan approach is used to assess the impacts of disruption in each link of the network. In order to appraise how significant a specific type of line is to network morphology, a scenario without a circular line is analysed. The final analysis examines the worst case scenarios resulting from a sequence of link disruptions.

\section{Methodology}

\subsection{Trip assignment to each network link}

The number of trips is obtained for each link of the network in order to incorporate the demand perspective into vulnerability analysis. Disruption to links used by a larger number of trips will evidently have a much greater impact on network operation. In fact, the importance of a link between two stations is represented not only by its location in the network and the availability of good alternatives, but also by the number of passengers it handles (Chen et al., 2007; Jenelius, 2009).

The number of trips of each link is calculated from the O-D trip matrix. First, the optimum routes between each pair of stations are obtained in terms of travel time. These routes are combined with the total number of trips between stations. An all-or-nothing approach is used as it is understood that all passengers would choose the shortest travel time route between their station of origin and their destination. Subsequently, the sum of the number of trips 
on all optimum routes passing through each link in the network is calculated to obtain the total number of trips along each link.

\subsection{Calculation of criticality and vulnerability}

\subsubsection{Criticality}

The methodology used to estimate the criticality level of each link is based on assessing the variation in serviceability. The importance of a link for the network to function properly will become apparent if, when it fails to be operative, it has a significant effect on the overall performance of the system (Jenelius, 2009). It was assumed that when a link is disrupted travel time remain constant for all other non-disrupted links. Increased or decreased congestion has no effects on the travel time of the non-disrupted links.

Link closure can have two types of consequences. In cases where there is no trip alternative (i.e. the network is subdivided into two parts), criticality can be measured in relation to the total number of trips unable to reach the destination station (unsatisfied demand). When an alternative is available (i.e. that do not subdivide the network into two parts), disruption of the link leads to an increase in user travel time because of the need to use routes that are not optimal.

In the second case, the criticality can be measured by analysing impacts on travel times. The weighted average travel time is used as a serviceability indicator. It was calculated as follows:

$\bar{T}_{i}=\frac{\sum_{j} T_{i j} F_{i j}}{F_{i}}$

where $\bar{T}_{i}$ is the average travel time for the station $i, T_{i j}$ is the travel time according to the fastest route from station $i$ to station $j, F_{i j}$ is the number of trips with origin in station $i$ and destination in station $j$, and $F_{i}=\sum_{j} F_{i j}$ is the total number of trips generated from station $i$.

The average overall travel time in the original scenario $\left(\bar{T}_{0}\right)$ were like:

$\bar{T}_{0}=\frac{\sum_{i} \sum_{j} T_{i j} F_{i j}}{\sum_{i} \sum_{j} F_{i j}}=\frac{\sum_{i} F_{i} \bar{T}_{i}}{\sum_{i} F_{i}}$

And the impact of disruption in each link $\left(\bar{T}_{a}\right)$ was assessed through the average times without each link, using:

$\bar{T}_{a}=\frac{\sum_{i} \sum_{j} T_{i j} F_{i j a}}{\sum_{i} \sum_{j} F_{i j}}=\frac{\sum_{j} F_{i} \bar{T}_{i a}}{\sum_{i} F_{i}}$

where $T_{i j a}$ is the travel time from station $i$ to station $j$ according to the fastest detour when link $a$ is disrupted.

Finally, average overall travel time without the link $a$ were compared with average overall travel time in the original scenario, using the equation:

$I_{a}=\bar{T}_{a}-\bar{T}_{0}$

where $I_{a}$ is the time lost by closure of link $a$. The process is repeated for each link. Results at link level are mapped. The importance (criticality) for those links with trip alternative (i.e. that do not subdivide the network into two parts) was obtained from this analysis.

\subsubsection{Station exposure}

Vulnerability was measured at the station level (station exposure). In stations affected by link disruptions without trip alternative, the station exposure was calculated as the number of links needed until a station with two or more trip alternatives is found.

Furthermore, in all stations, station exposure was calculated as the average time loss due to interruption for all links with trip alternative. Let $A$ be the set of all links in the network. $A_{w} \subseteq A$ is the subset of all links with route alternatives when disrupted. Exposure is calculated using:

$V_{i}=\frac{\sum_{a \in A_{w}}\left(\bar{T}_{i a}-\bar{T}_{i}\right)}{N_{w}}$

where $V_{i}$ is the exposure in station $i$, and $N_{w}$ is the number of links in $A_{w}$. An interpretation of station exposure is the expected average travel time increase for trips with origin in station $i$ when a randomly chosen link in the set $A_{w}$ is disrupted.

The average time loss of interruption for links with trip alternative was also analysed according to lines and types of line.

\subsubsection{Sequence of critical disruptions}

The more critical scenarios were identified in a sequence of link disruptions, applying the methodology used by Matisziw et al. (2009). These scenarios were obtained for those links with trip alternative. First, the link that has the greatest impact on times is identified. Then, losses caused by disruption of the rest of the links are recalculated, but now in a network in which the previous link has been eliminated. This process is repeated until the desired numbers of critical scenarios are identified (with one disruption, two disruptions, and so on). In this paper, the combinations with two, three, four and five most critical links have been identified. The impact of the most critical scenario has been compared with the average overall travel time for 10 random combinations of five disrupted links.

\subsubsection{Changes in trips distribution}

Finally, trip distribution is recalculated for three scenarios: without the most critical link, without the most critical sequence of five link disruptions and without five random links. This enables the identification of links absorbing trips that have been diverted because of disruption in different scenarios.

\section{Case study: Madrid Metro Network}

\subsection{Data}

The following cartographical and statistical information was used to analyse network vulnerability in the Madrid Metro system.

- The Madrid Metro Network, showing stations and links for the year 2007. For each link of the network, length and journey time are included. Sixty passageways are also incorporated to enable the simulation of transfers at stations with more than one line, with a time penalty for each transfer.

- OD trip matrix between stations in October 2007 (Madrid Regional Transport Consortium). This gives the total number of trips made between each pair of stations during a normal weekday. From this information, network flows distribution can be obtained. It is also used to calculate average times of the relations each station has with the rest of the stations, although these are weighted by the number of trips between each one.

Information has been treated and network analyses computed in ArcGIS-ArcINFO 10

\subsection{General characteristics of Madrid Metro Network}

In 2007 Madrid Metro consisted of 12 lines and 239 stations, with 268 links connecting the different stations. An essential element for reducing network vulnerability is the convergence of several lines at the same station. The Madrid Metro has 27 stations 


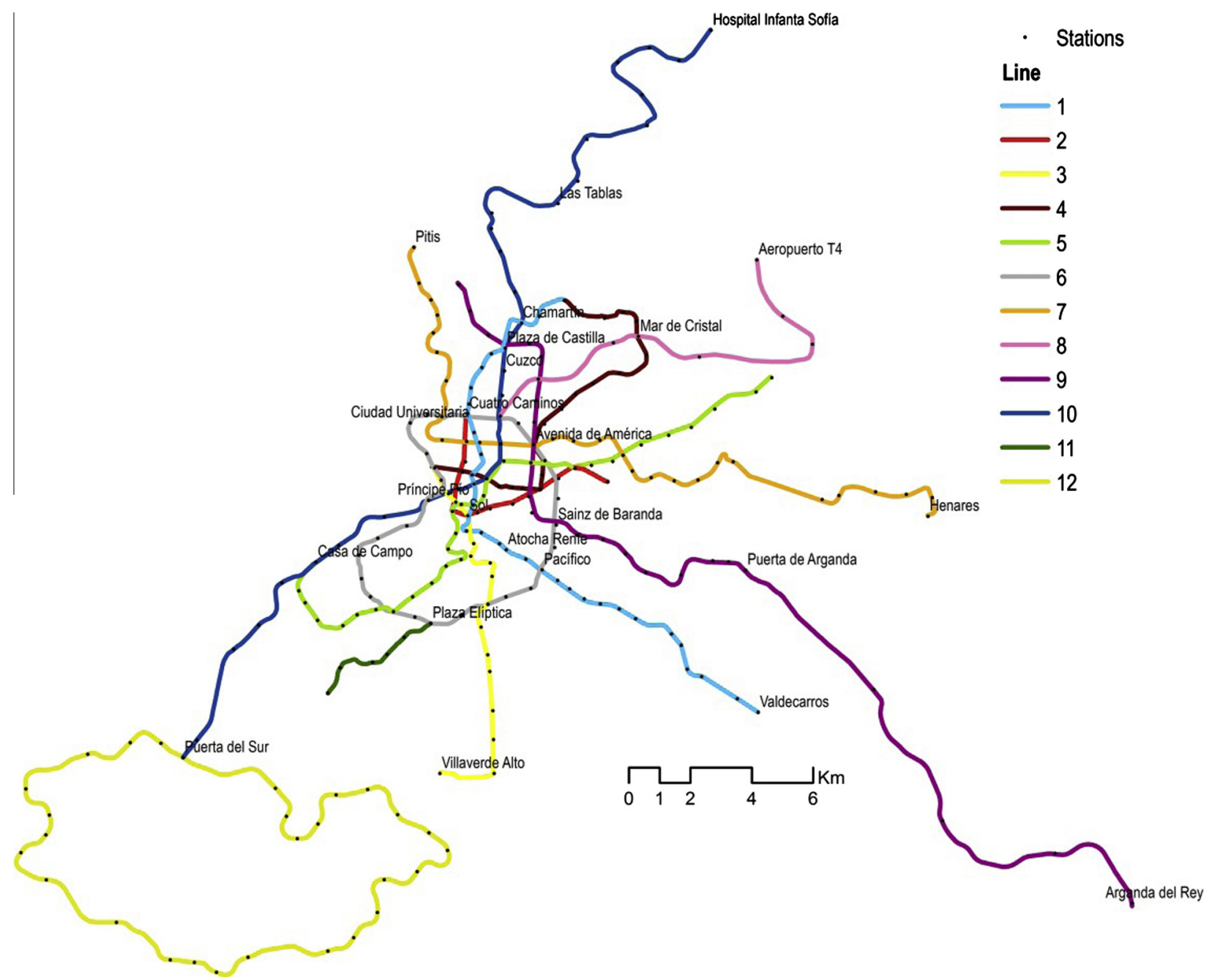

Fig. 1. Madrid Metro Network.

connecting 2 lines, 10 stations connecting 3 lines and 1 station connecting 4 lines. The network structure is complex and well developed, as is to be expected in one of the longest and most concentrated systems in the world. Of the 12 lines making up the network, 5 are radial (Lines 1, 2, 3, 5 and 10), converging at the centre of the system, 3 are transversal (Lines 4, 7 and 9) and do not pass through the centre, one is circular (Line 6), two are peripheral (Lines 8 and 11) and one is suburban (Line 12) (Fig. 1). Topological measures show the complexity of the Madrid Metro system in comparison with other Metro networks (see, for example, Criado et al., 2007; Derrible and Kennedy, 2010). Topo- logical data are similar to famous metros system like London, Moscow, Mexico or Tokyo (Table 1).

On a normal day in October 2007 almost 2,500,000 trips were made on the Madrid Metro. Line 10 and the circular Line 6 were those that channelled most trips, with 367,000 and 353,000 trips respectively, followed by other radial lines like Lines 1,3 and 5 (Table 2 and Fig. 2). Lines 6 and 10 are also the lines that have the greatest average number of trips per link. The maximum number of trips is found on Line 10, with links in the city centre supporting more than 250,000 trips. Peripheral and transversal lines carry a much smaller number of passengers.

Table 1

Basic topological indicators in different Metro networks.

\begin{tabular}{|c|c|c|c|c|c|}
\hline & Average distance $^{\mathrm{a}}(\mathrm{km})$ & Network diameter ${ }^{a}(\mathrm{~km})$ & Average degree $^{a}$ & Complexity $^{\mathrm{b}}$ & Degree of connectivity \\
\hline Madrid & 12.33 & 41 & 2.35 & 1.78 & 0.62 \\
\hline London & 15.54 & 38 & 2.05 & 1.87 & 0.64 \\
\hline Moscow & 12.32 & 37 & 2.11 & 1.60 & 0.56 \\
\hline Mexico & 10.94 & 29 & 2.22 & 1.34 & 0.47 \\
\hline Tokyo & 10.09 & 32 & 2.41 & 1.95 & 0.67 \\
\hline
\end{tabular}

a Data obtained from: Criado et al., 2007.

b Data obtained from: Derrible and Kennedy, 2010. 
Table 2

Total trips distribution, by links and lines (trips during a normal weekday).

\begin{tabular}{|c|c|c|c|c|c|c|}
\hline Line & $\mathrm{N}^{\circ}$ of links & Total trips in the line & Maximum & Minimum & Mean & Standard deviation \\
\hline All the network & 268 & $2,446,665$ & 261,821 & 2,267 & 58,756 & 53,368 \\
\hline 1 & 32 & 272,955 & 186,080 & 2,267 & 86,875 & 56,022 \\
\hline 2 & 15 & 57,414 & 37,525 & 10,790 & 19,889 & 8,548 \\
\hline 3 & 17 & 203,377 & 130,135 & 16,576 & 73,242 & 33,822 \\
\hline 4 & 22 & 120,320 & 79,611 & 12,671 & 40,709 & 18,343 \\
\hline 5 & 31 & 197,486 & 131,923 & 8,528 & 65,563 & 33,808 \\
\hline 6 & 28 & 353,394 & 213,262 & 88,673 & 140,131 & 41,690 \\
\hline 7 & 28 & 137,023 & 94,806 & 2,759 & 42,217 & 27,936 \\
\hline 8 & 7 & 162,657 & 105,331 & 11,777 & 57,326 & 32,329 \\
\hline 9 & 25 & 147,378 & 104,170 & 10,355 & 43,207 & 23,972 \\
\hline 10 & 30 & 367,062 & 261,821 & 4,209 & 105,240 & 88,563 \\
\hline 11 & 5 & 33,402 & 21,105 & 4,134 & 12,296 & 5,868 \\
\hline 12 (MetroSur) & 28 & 73,619 & 43,720 & 18,769 & 29,899 & 6,658 \\
\hline
\end{tabular}

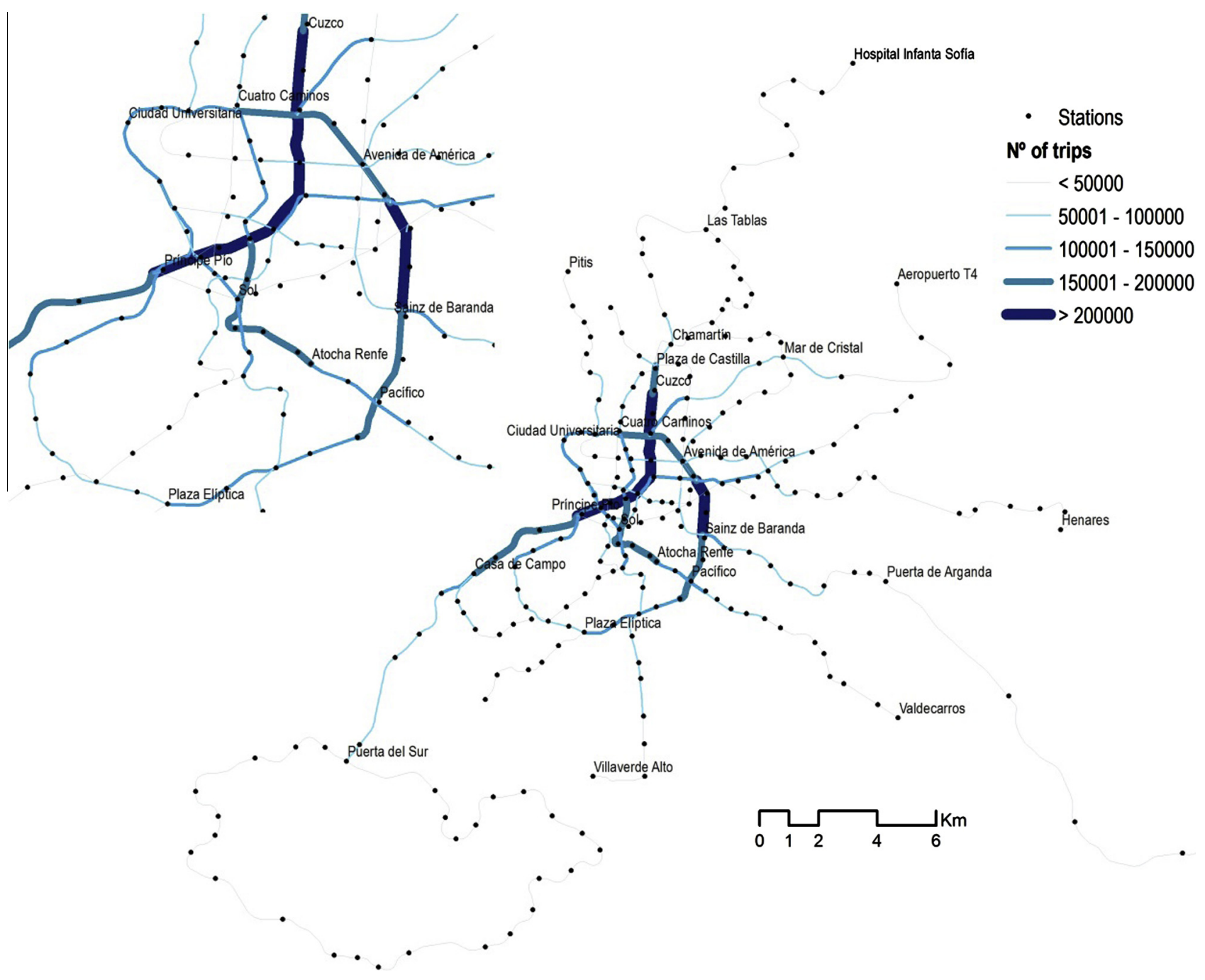

Fig. 2. Number of trips during a normal weekday by each link in the network.

\section{Results}

\subsection{Links without travel alternatives: unsatisfied demand}

Fig. 3 shows the number of trips that could not be undertaken for those links with no alternative trip options. These are links connecting areas on the outskirts of the city with the rest of the network. The most critical links affect the connection of long lines (leaving many links disconnected) that serve the densely populated residential areas to the south of the city: on Line 1 $(116,000$ missed trips), Line $9(104,000)$ and Line $10(100,000)$ (Table 3 ). In the case of links on Line 10, disruption leaves the suburban Line 12 with no connection to the rest of the network. In other cases, disruption has important consequences for certain 


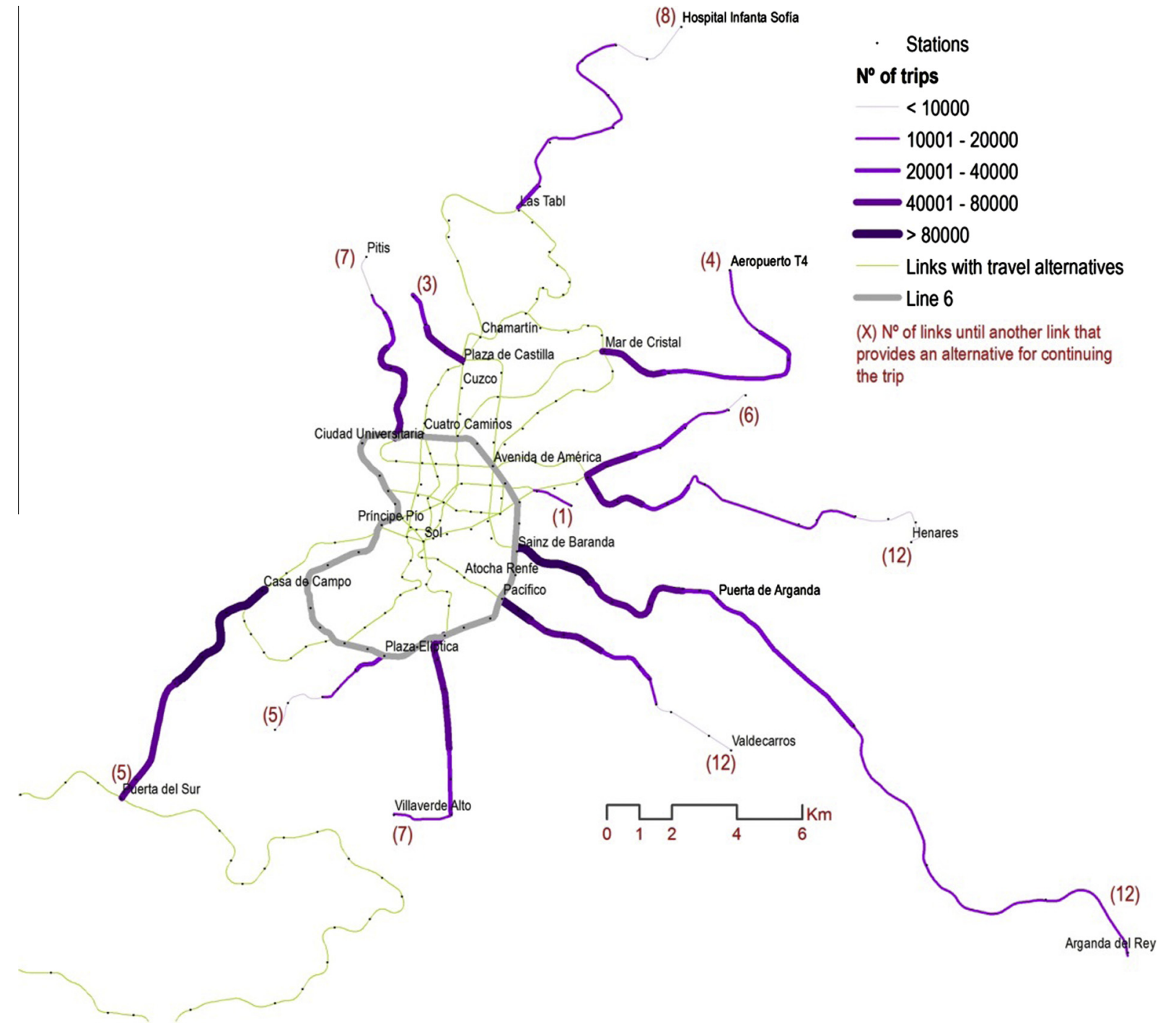

Fig. 3. Number of trips during a normal weekday by links without travel alternatives.

Table 3

Number of links without travel alternatives and trips during a normal weekday in these links, by line.

\begin{tabular}{clllll}
\hline Line & Number of links & Minimum & Maximum & Mean & SD \\
\hline 1 & 12 & 2,267 & 115,888 & 46,271 & 36,798 \\
2 & 1 & 15,501 & 15,501 & 15,501 & 0 \\
3 & 7 & 16,576 & 72,801 & 46,753 & 21,204 \\
5 & 6 & 8,528 & 76,282 & 35,337 & 25,529 \\
7 & 19 & 2,759 & 77,769 & 30,450 & 23,230 \\
8 & 4 & 11,777 & 53,160 & 31,859 & 17,713 \\
9 & 15 & 10,355 & 104,170 & 47,139 & 30,078 \\
10 & $13+(28$ from line 12) & 4,209 & 100,145 & 39,142 & 35,019 \\
11 & 5 & 4,134 & 21,105 & 12,297 & 6,562 \\
\hline
\end{tabular}

elements of the city. For example, disruptions to links of Line 8 leave the Airport and the Exhibition Centre without service. Fig. 3 shows the importance of the circular lines for network robustness (Derrible and Kennedy, 2010). Circular Line 6 provides an alternative route on five of the twelve lines that enter the city centre.

The degree of exposure at station level was calculated according to the number of links between the station and the closest link that provides an alternative for continuing the trip. The most vulnerable stations are the terminals of lines that have successions of unconnected links (Fig. 3).

\subsection{Links with travel alternative: time loss}

The average of the weighted average travel times in the normal situation is $30.47 \mathrm{~min}$ (Eq. (2)). Time loss resulting from the disruption of one of the links averages $0.5 \mathrm{~min}$, meaning $1.7 \%$ in global time. However, some links have a high criticality level. The link where most time is lost (2.2 min) shows a $7.2 \%$ loss in global travel times (Table 4). The most critical links are located on Lines 10 and 6 (Table 5). High values also appear on some links of Lines 1 and 12 (losses above $4 \%$ ). Logically, the most vulnerable lines are those

Table 4

Weighted average travel times (minutes) for disruption of each link of the network.

\begin{tabular}{lllll}
\hline & Minimum & Maximum & Mean & Standard Deviation \\
\hline Time averages & 30.12 & 32.66 & 30.47 & 0.39 \\
Losses & 0.01 & 2.19 & 0.51 & 0.39 \\
Percentage losses (\%) & 0.05 & 7.20 & 1.67 & 1.30
\end{tabular}

* Only disruptions of links with travel alternatives are included. 
Table 5

Increases in weighted average travel times," by lines.

\begin{tabular}{|c|c|c|c|c|c|c|c|c|c|}
\hline \multirow[t]{2}{*}{ Line } & \multirow[t]{2}{*}{$\mathrm{N}^{\circ}$ of links } & \multicolumn{4}{|c|}{ Total losses (minutes) } & \multicolumn{4}{|c|}{ Percentage losses } \\
\hline & & Minimum & Maximum & Mean & SD & Minimum & Maximum & Mean & SD \\
\hline 1 & 20 & 0.1 & 1.5 & 0.7 & 0.4 & 0.31 & 4.80 & 2.41 & 1.34 \\
\hline 2 & 14 & 0.1 & 0.3 & 0.2 & 0.1 & 0.13 & 1.12 & 0.50 & 0.32 \\
\hline 3 & 10 & 0.1 & 0.7 & 0.4 & 0.2 & 0.05 & 2.27 & 1.28 & 0.81 \\
\hline 4 & 22 & 0.1 & 0.7 & 0.2 & 0.2 & 0.18 & 2.39 & 0.73 & 0.53 \\
\hline 5 & 25 & 0.2 & 0.9 & 0.4 & 0.2 & 0.66 & 2.98 & 1.32 & 0.52 \\
\hline 6 & 28 & 0.4 & 1.7 & 0.8 & 0.4 & 1.20 & 5.48 & 2.59 & 1.20 \\
\hline 7 & 9 & 0.1 & 0.5 & 0.3 & 0.1 & 0.37 & 1.59 & 0.89 & 0.43 \\
\hline 8 & 3 & 0.6 & 0.7 & 0.7 & 0.0 & 2.09 & 2.41 & 2.27 & 0.16 \\
\hline 9 & 10 & 0.1 & 0.2 & 0.2 & 0.0 & 0.46 & 0.71 & 0.58 & 0.09 \\
\hline 10 & 17 & 0.2 & 2.2 & 0.9 & 0.7 & 0.50 & 7.17 & 2.81 & 2.18 \\
\hline 12 & 28 & 0.4 & 1.3 & 0.8 & 0.2 & 1.41 & 4.27 & 2.58 & 0.80 \\
\hline
\end{tabular}

* Only disruptions of links with travel alternatives are included.

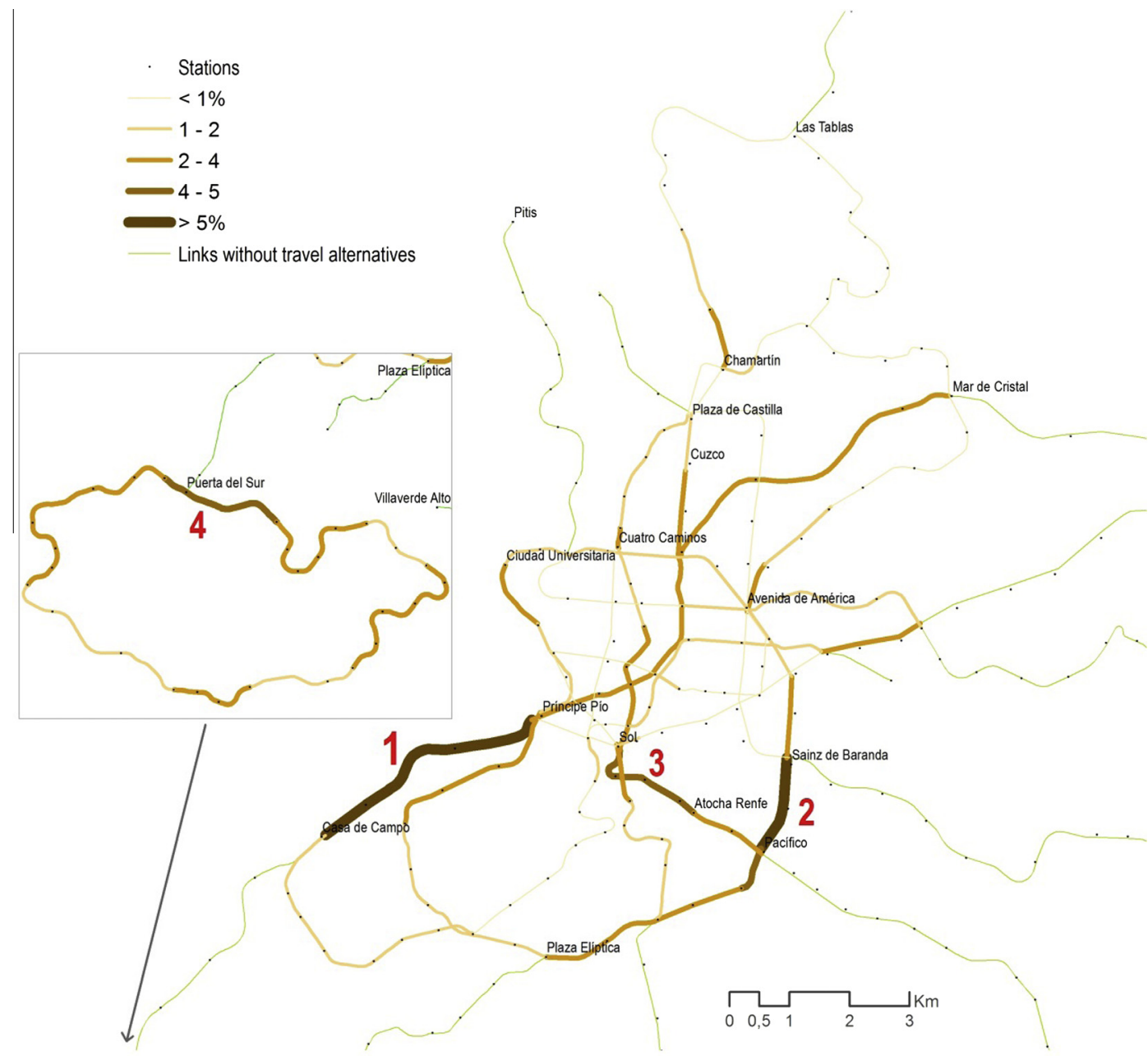

Fig. 4. Impact of the disruption of each link on global weighted average travel times (percentage change).

that carry a high number of trips but also those affected by significant detours.

Fig. 4 shows the distribution of percentage losses in weighted average travel times for disruption in each link of the network (criticality). The criticality of a link depends on its position in the network and on the total number of trips channelled through it. The most critical links are located in areas of connection between the outlying residential zones and the city centre. These links sup- port a considerable amount of trips and disruption implies significant detours.

Time losses are between $6.7 \%$ and $7.2 \%$ in zone 1 . These links connect the large suburban municipalities served by Line 12 and the densely populated areas to the south west of Madrid with the city centre. Around 160,000 trips a day pass through these links (Fig. 1). In cases of disruption, these trips have to be diverted an extra journey of at least 15 stations. The next most critical situation 
Table 6

Losses in weighted average travel times," by types of line.

\begin{tabular}{lll}
\hline Type of line & Lines & Absolute difference in minutes (average for all links on the line) \\
\hline Radial & $1,2,3,5$ and 10 & 0.51 \\
Transversal & $4,7,9$ & 0.22 \\
Circular & 6 & 0.79 \\
Orbital & 8,11 & 0.37 \\
Suburban Circular & 12 & 0.79 \\
\hline
\end{tabular}

Only disruptions of links with travel alternatives are included.

Table 7

Data on weighted average travel times of stations with and without some of the major lines of the network.

\begin{tabular}{|c|c|c|c|c|c|}
\hline Line & Statistic & Scenario with this line (Minutes) & Scenario without this line (Minutes) & Difference (Minutes) & Percentage difference (\%) \\
\hline \multirow[t]{3}{*}{ Line 6} & Mean & 30.69 & 32.09 & 1.40 & 4.6 \\
\hline & Standard Deviation & 18.86 & 20.25 & 1.39 & 7.4 \\
\hline & Coefficient. of Variation & 61.45 & 63.1 & 1.65 & 2.7 \\
\hline \multirow[t]{3}{*}{ Line 10} & Mean & 30.32 & 31.63 & 1.31 & 4.3 \\
\hline & Standard Deviation & 17.29 & 18.25 & 0.96 & 5.5 \\
\hline & Coefficient. of Variation & 57.03 & 57.70 & 0.67 & 1.2 \\
\hline \multirow[t]{3}{*}{ Line 1} & Mean & 31.06 & 31.65 & 0.59 & 1.9 \\
\hline & Standard Deviation & 18.6 & 18.94 & 0.34 & 1.8 \\
\hline & Coefficient. of Variation & 59.88 & 59.84 & -0.04 & -0.07 \\
\hline \multirow[t]{3}{*}{ Line 5} & Mean & 29.87 & 30.15 & 0.28 & 0.9 \\
\hline & Standard Deviation & 18.34 & 18.54 & 0.20 & 1.1 \\
\hline & Coefficient. of Variation & 61.40 & 61.49 & 0.09 & 0.1 \\
\hline
\end{tabular}

In order to compare two scenarios, with and without the lines considered, stations on lines without a connection to a different line have been eliminated from both scenarios (as a consequence they would be without service).

Table 8

Weighted average travel times (minutes) at station level as a result of disruption in each links with travel alternatives.

\begin{tabular}{lrlr}
\hline & Mean & Standard deviation & Maximum \\
\hline Station times with disruption to links & 33.37 & 7.02 & 21.30 \\
Station times (normal situation) & 32.79 & 7.13 & 20.76 \\
Total loss & 0.57 & 0.42 & 0.07 \\
Percentage loss & 1.90 & 1.57 & 61.88 \\
\hline
\end{tabular}

involves two links of Line 6 (Zone 2), with impacts above $5 \%$ in global time. Disruption to these links affects some 190,000 trips between the south of the city and the east, making it necessary to travel into the city centre (Sol station) in order to travel out again to the east. Two other critical zones, with losses of over $4 \%$, are found in the city centre on Line 1 (Zone 3 ) and on the outskirts of the city, the links connecting the suburban circular Line 12 with Line 10 , which gives access to the rest of the network (Zone 4).

Given the importance of circular lines for network robustness (Derrible and Kennedy, 2010), the role of the circular Line 6 in the Madrid Metro was analysed. Average travel times were compared for two scenarios: with and without circular Line 6. This with/without analysis was also carried out for some of the major radial lines in order to compare the effects with those of removing the circular line.

In Madrid Metro Network, circular lines are the most critical ones (Table 6), with average losses in their links of $2.6 \%$. In contrast, radial and transversal lines are much less vulnerable. At the same time, Line 6 plays a crucial role in network robustness as it provides travel alternatives on numerous occasions. In order to show its importance, travel times were been calculated using different scenarios: with and without Line 6 , and with and without three of the most important radial lines in the network. The data show improved travel times produced by these lines compared with the rest of the stations making up the network (Table 7). Line 6 gives improved travel times of $4.6 \%$ for the rest of the lines, a much higher value than radial lines such as Lines 1 and 5 . Only Line 10 , which integrates a large part of the north-south relations in the city centre, produces similar improvements. Line 6 also reduces differences in station accessibility by improving the situation of stations where accessibility is poor (those on the periphery), as shown by changes in the coefficient of variation.

Table 8 shows average travel times at station level (Eq. (5)), during both normal operation and in a situation of disruption in each link of the network. Average station loss is $0.6 \mathrm{~min}$, with a maximum of almost $2 \mathrm{~min}$. This means average percentage losses of almost $2 \%$, with a maximum of $7 \%$. The most exposed stations are generally in the south of the city (Fig. 5). Suburban Line 12 is particularly exposed, with losses of over $5 \%$ in all its stations. Stations in the north and centre of the city are less exposed and are able to maintain service and accessibility at levels approaching the original ones. The morphology and density of the network is a key factor as greater density in these areas makes travel easier and provides alternative routes.

\subsection{The most critical disruption sequences: simulating a targeted attack}

Fig. 6 shows the worst case scenarios arising from a sequence of disruptions to links of the Madrid Metro system. The link that produces the worst scenario in the case of disruption is between Lago and Príncipe Pío stations (Line 10). If a second disruption occurs, 


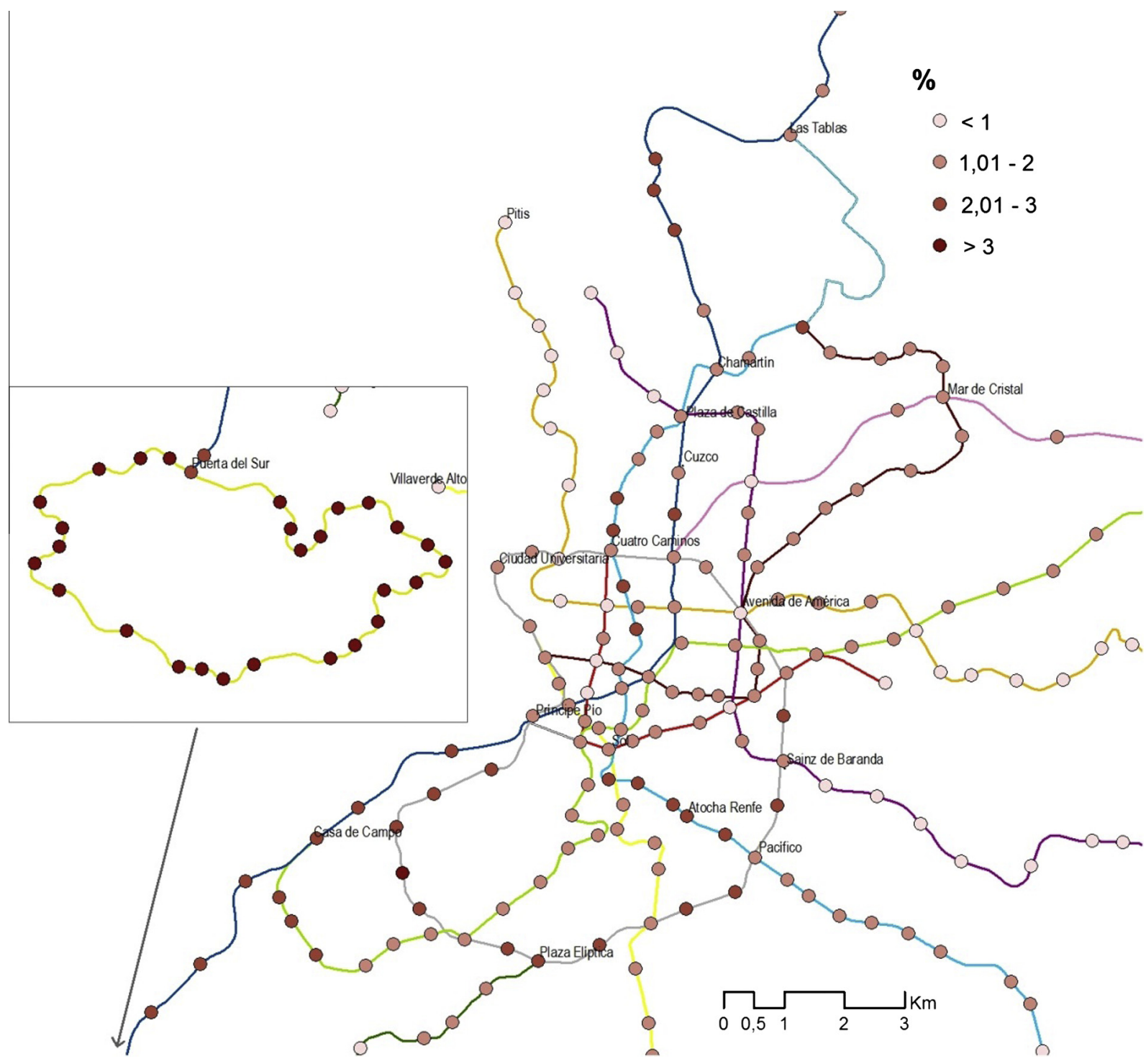

Fig. 5. Average percentage losses by station with disruption in links with trip alternatives (station exposure).

the most critical link (in a network in which the previous link has been eliminated) is located on Line 6. Disruption to these two links results in an increase in average global times of $4.1 \mathrm{~min}$, an increment of $13.5 \%$ (Table 9). Fig. 6 shows the most critical situations with disruptions in three, four and five links. In the worst scenario, with disruption in five links, average times increase by $13 \mathrm{~min}$ (around 43.6\%). It is significant that these are all located in the south of the network. The total number of trips requiring a change of route as a result of this sequence of disruptions rises from 163,000 when the first link is disrupted (around $6.6 \%$ of total trips) to 753,000 when disruption occurs in five links (around 31\%). If an attack on the network takes place on five random links, there is a much lower time loss (around 19.1\%), in a situation in which more than $22 \%$ of the trips would have to be modified (Table 9).

\subsection{Redistribution of trip flows}

Fig. 7 shows trips redistribution for three different scenarios: disruption of the most critical link (Fig. 7a); a targeted attack on the 5 links that would produce the most critical situation (7b); and an attack on 5 random links (7c). Although a good number of these trips would be channelled through other public transport networks, such as buses or suburban trains, all the trips have been redistributed in order to ascertain which links would be the most affected as a result of them being diverted to other lines. With disruption of the most critical link, the trips towards the city centre now passes to Line 5 until reaching the circular Line 6 , which serves as a redistribution point for reconnecting with Line 10 and, to a lesser extent, to Lines 3 and 1 (Fig. 7a). As a result of this redistribution, links of Line 5 would multiply their current number of trips by 4 , and those of Line 6 by 2 . In contrast, the number of trips on the central links of Line 10 would drop by between $10 \%$ and $20 \%$. In scenarios with 5 disruption links, redistribution is more complex, but in both cases, Line 6 once more plays a crucial role in trip redistribution. In the targeted attack scenario, links on the southern part of Line 6 would redistribute trips coming into the city centre, which would use Line 6 in order to reach Line 5 to access the centre. The number of trips in the links of Lines 6 and 5 would be multiplied by 10 , thus producing saturation. In the case of the 5 random closures, links of the NW and NE of Line 6 receive the greatest number of trips, duplicating their normal demand. However, in this case the trips redistribution between different lines is much more homogeneous. 

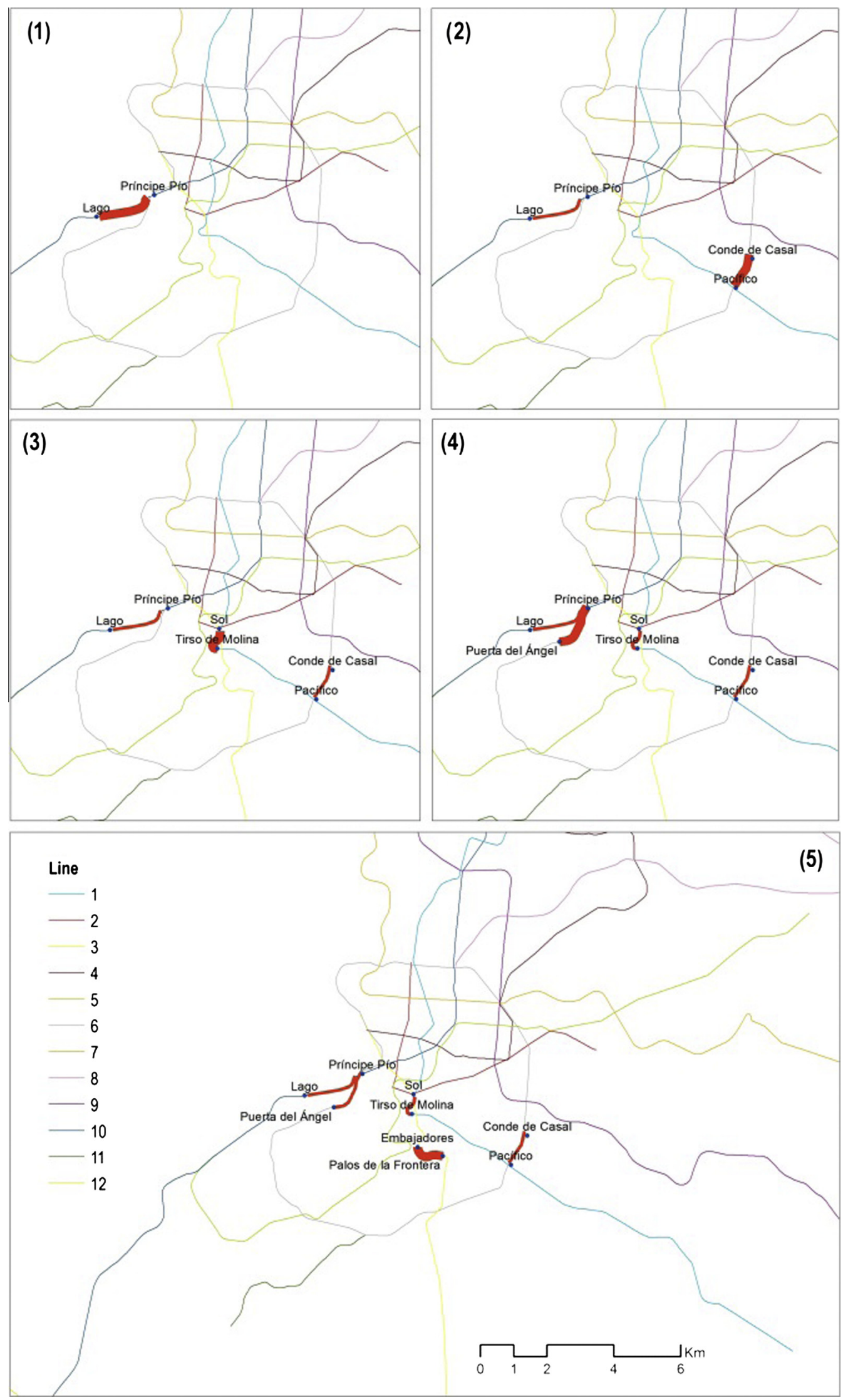

Fig. 6. Links in a sequence of more critical successive disruptions (only for links with trip alternative). 
Table 9

Weighted average travel times (minutes) and trips affected in the most critical disruption sequences."

\begin{tabular}{|c|c|c|c|c|c|}
\hline Critical disruption sequences & Average times (minutes) & Accumulated losses (minutes) & Accumulated losses (\%) & Total trips affected & $\%$ of the total \\
\hline Initial situation & 30.47 & & & $2,446,665$ & \\
\hline 1 (Lago-P. Pío; Line 10) & 32.66 & 2.19 & 7.2 & 162,623 & 6.6 \\
\hline 2 (Pacífico-C. Casal; Line 6) & 34.57 & 4.10 & 13.5 & 357,394 & 14.6 \\
\hline 3 (Tirso de Molina-Sol; Line 1 ) & 37.39 & 6.92 & 22.7 & 532,973 & 21.8 \\
\hline 4 (Pta. Ángel-P. Pío; Line 6) & 39.55 & 9.08 & 29.8 & 657,908 & 26.9 \\
\hline 5 (P. Frontera-Embajadores; Line 3) & 43.74 & 13.27 & 43.6 & 752,592 & 30.8 \\
\hline Random attack (on 5 links) & 36.30 & 5.83 & 19.1 & 541,782 & 22.1 \\
\hline
\end{tabular}

**Average value of 10 combinations of five random disrupted links.

"The most critical links and "Random attack" have been selected excluding links that would lead to unsatisfied demand.

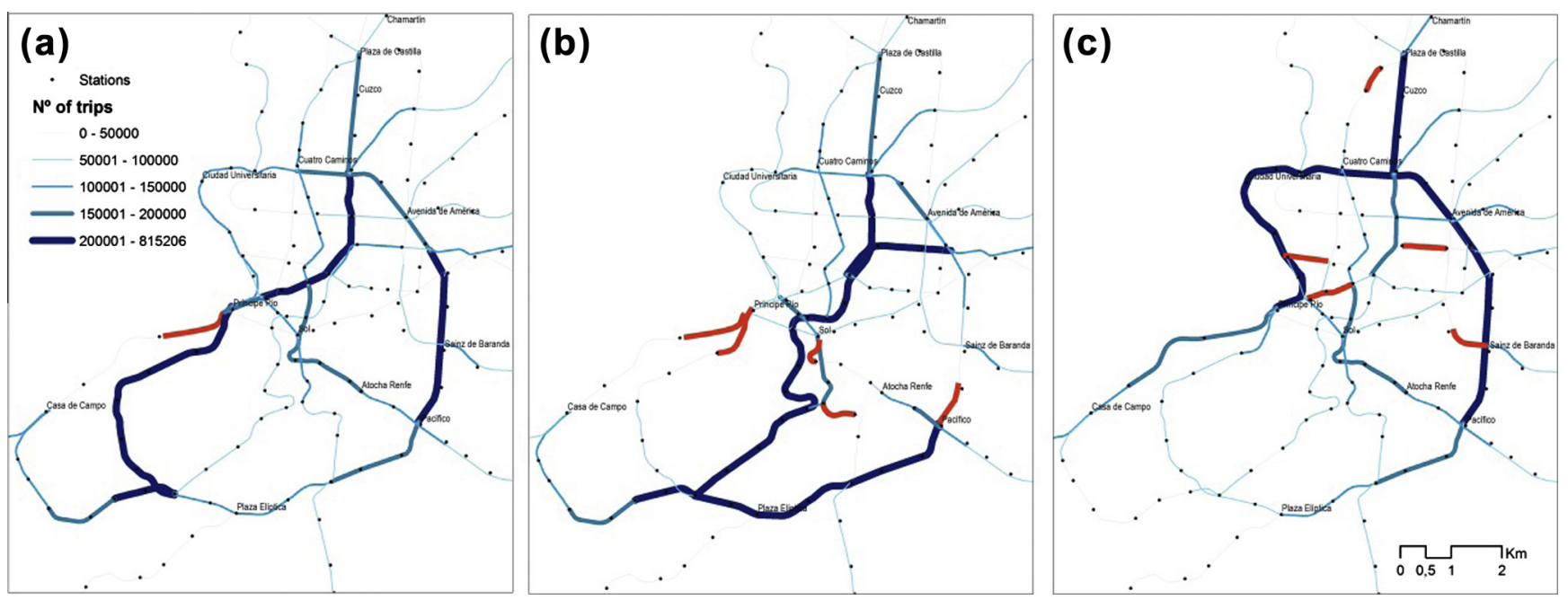

Fig. 7. Trips redistribution in three scenarios (Closed links in (c) (example obtained by first random selection): Ibiza-Sainz de Baranda (L9), Tetuán-Valdeacederas (L1), Arguelles-San Bernardo (L4), Plaza de España-Tribunal (L10), Rubén Darío-Núñez de Balboa (L10).)

\section{Conclusions}

In this paper we present a methodology for analysing the criticality and vulnerability of a public transport network. In contrast with studies based on graph theory indicators, we have used real network trips distribution and the impact on trips of disruption on links of the network is analysed. A full scan approach is used to analyse the impact that each of the links has on network relations as a whole (criticality). This methodology has been automated in a GIS, enabling the most critical links and lines to be identified. Station exposure has been obtained from data on criticality. This not only enables the distribution of criticality and vulnerability to be known, but also assesses possible scenarios (such as the role of Line 6 ) and obtains the most critical scenarios in a sequence of link disruptions. The automation of processes also makes it easier to identify critical disruption sequences in the links and trips redistribution in the different scenarios. It is essential to have this information available in order to mitigate the effects of incidents on the network and to plan new lines or extend existing ones in such a way that vulnerability and critical elements are reduced.

The effect of closure is different for each link and depends on its position in the network and the total number of trips channelled through it. When no trip alternative is available, disruption leaves the link without any possibility of service and the use of other transport networks is required. These situations occur in the radial lines that access the city. The greatest criticality is found in links of lines that connect a large number of stations and have high passenger numbers. With respect to links with an alternative route, the most critical are those that channel a significant number of trips in areas where network density is lower and there are few alternatives for these trips, resulting in long detours. In contrast, in areas where network density is high, trips are redistributed along a greater number of lines and passengers find alternatives to the disrupted links that are acceptable in terms of time.

The exposure of the stations has been obtained from data on criticality. The most exposure are the ones situated along the most critical links in the south of the city, while stations in the north and centre of the city are less exposure. The morphology and density of the network is a key factor. In this respect, this paper draws attention to the importance of circular lines as an element of network robustness. By generating trip alternatives, these lines enable the impacts of incidents in different links of the network to be absorbed.

Finally, with the model developed it is possible to obtain critical sequences in disrupted links of the network, simulating a possible targeted attack or act of sabotage. Analysis of the most critical scenarios reveals that the impact is much greater in the targeted attack than in a random attack, with time losses of up to $24 \%$ more, but it also shows much greater congestion on lines affected by diverted trips.

One element that has not been considered concerns the characteristics of the population affected when a link is disrupted. Vulnerability will be greater in stations located in the poorer residential areas where people depend more on public transport. In Madrid, the most critical links and the most vulnerable stations on the Metro network are located in the south, which is the poorest sector of the city. By taking the characteristics of the affected population into account, a relation between network vulnerability 
analysis and social vulnerability can be established, although this has not been included in this study.

Vulnerability changes in the system depending on the time of day were not considered. Whilst our results reflect the average situation along the day, there are fewer impacts and less vulnerability in the late evening versus rush-hours. It was also assumed that when a link is disrupted travel time remains constant for all other non-disrupted links. Nevertheless, in the short-term it is possible to expect increases in travel times as a result of congestion on non-disrupted links. On the other hand, in the long-term, travel delays could potentially alter the general travel patterns. These changes may remain even when the normal service has been re-established.

Our approach considers the demand to be inelastic (similar to Jenelius et al., 2006), thus the demand remains constant with disruption links. Another line for future study deriving from this paper is the consideration of trip alternatives that include the use of other public transport networks. As public transport operates as a multimodal network, many of the incidents occurring on the Metro are resolved by passengers finding alternative networks. Madrid has a particularly dense network of urban and interurban buses and, within the city itself, a further alternative for trips can often be found in the suburban train service. However, in this paper the Metro system is not studied as part of a wider multimodal network. Instead, it has been studied separately in order to assess the role played by its own elements with respect to its vulnerability.

However, results shown in this paper provide extremely useful information for mitigating the effects of potential incidents on the network. For example, identifying the most critical links and linksequences facilitates control and management tasks, which eventually increments security. Also, knowing the redistribution of flows on the network and the affected lines is of great help to identify and manage new needs as well as a potential increase in the frequency or capacity of those lines. In the case of an incident over a link with no alternative route, identifying unsatisfied demand gives useful information of the trips that would be transferred to other public transport networks.

The results obtained are also of high interest in planning new lines or broadening the network. New criteria for vulnerability and critical element reduction could be introduced in addition to current efficiency and equity criteria when designing new lines and deciding the location of new stations. In this regard, circular lines play a key role due to their capacity to provide multiple alternative routes, and thus enabling an efficient way to redistribute flows.

This study has been funded by the Ministerio de Economía y Competitividad (SPILLTRANS Project, TRA2011-27095) and the Programa de Formación del Profesorado Universitario del Ministerio de Educación y Cultura. We also want to express our gratitude to the anonymous referees for their comments and suggestions which have helped us to improve the paper.

Albert, R., Hawoong, J., Barabási, A.L., 2000. Error and attack tolerance of complex networks. Nature 406, 378-382.

Albert, R., Albert, I., Nakarado, G.L., 2004. Structural vulnerability of the North American power grid. Phys. Rev. E 69 (025103), 1-4.

Alderson, D.L., Brown, G.G., Carlyle, M.W., Anthony Cox, L., 2013. Sometimes There Is No "Most-Vital" Arc: assessing and Improving the operational resilience of systems. Military Operat. Res. 18 (1), 21-37.

Angeloudis, P., Fisk, D., 2006. Large subway systems as complex networks. Physica A 367, 553-558.

Bell, M.G.H., 2000. A game theory approach to measuring the performance reliability of transport networks. Transport. Res. B 34, 533-545.
Bell, M.G.H., Kanturska, U., Schmöcker, J.-D., Fonzone, A., 2008. Attacker-defender models and road network vulnerability. Philos. Trans. Royal Soc. A 366, $1893-$ 1906.

Berdica, K., 2002. An introduction to road vulnerability: what has been done, is done and should be done. Transport Policy 9, 117-127.

Berdica, K., Mattsson, L.G., 2007. Vulnerability: a model-based case study of the road network in Stockholm. In: Murray, A.T., Grubesic, T.H. (Eds.), Critical Infrastructure: Reliability and Vulnerability. Springer, New York, pp. 81-106.

Bono, F., Gutiérrez, E., 2011. A network-based analysis of the impact of structural damage on urban accessibility following a disaster: the case of the seismically damaged Port Au Prince and Carrefour urban road networks. J. Transport Geogr. $19,1443-1455$

Cats, O., Jenelius, E., 2012. Vulnerability analysis of public transport networks: A dynamic approach and case study for Stockholm. In: Proceedings of the 5th International Symposium on Transportation Network Reliability (INSTR2012), 535-544. Disponible en: <http://home.abe.kth.se/ jenelius/>.

Chang, S.E., 2003. Transportation planning for disasters: an accessibility approach. Environ. Plan. A 35, 1051-1072.

Chen, A., Yang, C., Kongsomsaksakul, S., Lee, M., 2007. Network-based accessibility measures for vulnerability analysis of degradable transportation networks. Networks Spatial Econ. 7 (3), 241-256.

Chen, B.Y., Lam, W.H.K., Sumalee, A., Li, Q., Li, Z., 2012. Vulnerability analysis for large-scale and congested road networks with demand uncertainty. Transport. Res. A 46 (3), 501-516.

Criado, R., Flores, J., Hernández-Bermejo, B., Pello, J., Romance, M., 2004. Effective measurement of network vulnerability under random and intentional attacks. J. Math. Modell. Algorithms 4, 307-316.

Criado, R., Hernández-Bermejo, B., Romance, M., 2007. Efficiency, vulnerability and cost: an overview with applications to subway networks worldwide. Int. J. Bifurcat. Chaos Appl. Sci. Eng. 17 (7), 2289-2301.

Derrible, S., Kennedy, C., 2010. Characterizing metro networks: state, form, and structure. Transportation 37, 275-297.

Erath, A., Birdsall, J., Axhausen, K.W., Hajdin, R., 2008. Vulnerability assessment of the Swiss road network. Research Report. Eidgenössische Technische Hochschule, Institut für Verkehrsplanung und Transportsysteme (ETH-IVT), Zurich.

Fang, Z., Shaw, S.L., Tu, W., Li, Q., Li, Y., 2012. Spatiotemporal analysis of critical transportation links based on time geographic concepts: a case study of critical bridges in Wuhan, China. J. Transport Geogr. 23, 44-59.

Garrison, W.L., 1960. Connectivity of the interstate highway system. Papers Reg. Sci. $6,121-137$.

Holme, P., Kim, B.J., Yoon, C.N., Hee, S.K., 2002. Attack vulnerability of complex networks. Phys. Rev. E 65 (056109), 1-14.

Jen, E., 2005. Robust design. In: Santa Fe Institute studies in Science of Complexity, Proceedings. Oxford University Press, NY.

Jenelius, E., 2009. Network structure and travel patterns: Explaining the geographical disparities of road network vulnerability. J. Transport Geogr. 17 (3), 234-244.

Jenelius, E., 2010. User inequity implications of road network vulnerability. J. Transport Land Use 2 (3-4), 57-73.

Jenelius, E., Mattsson, L.-G., 2005. Developing a methodology for road network vulnerability analysis. In: Nectar Cluster 1 Seminar, 12th - 13th May 2006 Molde University College, Molde (Norway).

Jenelius, E., Mattsson, L.-G., 2012. Road network vulnerability analysis of areacovering disruptions: a grid-based approach with case study. Transport. Res. A 46 (5), 746-760

Jenelius, E., Petersen, T., Mattsson, L.G., 2006. Importance and exposure in road network vulnerability analysis. Transport. Res. A 40 (7), 537-560.

Jenelius, E., Mattsson, L.-G., Levinson, D., 2011. Traveler delay costs and value of time with trip chains, flexible activity scheduling and information. Transport. Res. B 45 (5), 789-807.

Johansson, J., Jönsson, H., Johansson, H., 2007. Analysing the vulnerability of electric distribution systems: a step towards incorporating the societal consequences of disruptions. Int. J. Emergency Manage. 4 (1), 4-17.

Knoop, V., van Zuylen, H., Hoogendoorn, S., 2008. The influence of spillback modeling when assessing consequences of blockings in a road network. Eur. J. Transport. Infrastruct. Res. 8, 287-300.

Lou, Y., Zhang, L., 2011. Defending transportation networks against random and targeted attacks. J. Transport. Res. Rec. 2234, 31-40.

Masiero, L., Maggi, R., 2012. Estimation of indirect cost and evaluation of protective measures for infrastructure vulnerability: a case study on the transalpine transport corridor. Transport Policy 20, 13-21.

Matisziw, T.C., Murray, A.T., Grubesic, T.H., 2009. Exploring the vulnerability of network infrastructure to disruption. Ann. Reg. Sci. 43 (2), 307-321.

Matisziw, T.C., Murray, A.T., Grubesic, T.H., 2010. Strategic network restoration. Networks Spatial Econ. 10 (3), 345-361.

Matisziw, T.C., Grubesic, T.H., Guo, J., 2012. Robustness elasticity in complex networks. PLoS ONE 7 (7), e39788. http://dx.doi.org/10.1371/ journal.pone.0039788.

Mishra, S., Welch, T.F., Jha, M.K., 2012. Performance indicators for public transit connectivity in multi-modal transportation networks. Transport. Res. A 46 (7) 1066-1085.

Mouronte, M.L., Benito, R.M.a., 2012. Structural properties of urban bus and subway networks of Madrid. Networks Heterogeneous Media 7 (3), 415-428.

Murray, A., Grubesic, T., 2007. Critical Infrastructure. Reliability and Vulnerability. Springer, Berlin. 
Murray, A.T., Matisziw, T.C., Grubesic, T.H., 2008. A methodological overview of network vulnerability analysis. Growth Change 39, 573-592.

Myung, Y.S., Kim, H.J., 2004. A cutting plane algorithm for computing $<\mathrm{i}>\mathrm{k}<\mathrm{i}>$-edge survivability of a network. Eur. J. Oper. Res. 156 (3), 579-589.

Nurre, SG., Sharkey, TC., 2010. Restoring infrastructure systems: an integrated network design and scheduling problem. In: En Proceedings of the 2010 Industrial Engineering Research Conference.

Ratliff, H.D., Sicilia, G.T., Lubore, S.H., 1975. Finding the n most vital links in flow networks. Manage. Sci. 21, 531-539.

Reggiani, A., 2013. Network resilience for transport security: some methodological considerations. Transport Policy 28, 63-68.

Scott, D.M., Novak, D.C., Aultman-Hall, L., Feng, G., 2006. Network Robustness Index: a new method for identifying critical links and evaluating the performance of transportation networks. J. Transport Geogr. 14 (3), 215-227.

Sohn, J., 2006. Evaluating the significance of highway network links under the flood damage: an accessibility approach. Transport. Res. Part A 40, 491-506.
Suárez, P., Anderson, W., Mahal, V., Lakshmanan, T.R., 2005. Impacts of flooding and climate change on urban transportation: a systemwide performance assessment of the boston metro area. Transport. Res. D 10, 231-244.

Taylor, M.A.P., Susilawati, S., 2012. Remoteness and accessibility in the vulnerability analysis of regional road networks. Transport. Res. A 46, 761-771.

Taylor, M.A.P., Sekhar, S.V.C., D’Este, G.M., 2006. Application of accessibility based methods for vulnerability analysis of strategic road networks. Networks Spatial Econ. 6, 267-291.

von Ferber, C., Berche, B., Holovatch, T., Holovatch, Y., 2012. A tale of two cities: vulnerabilities of the London and Paris transit networks. J. Transport. Security 5 (3), 199-216.

Watling, D., Balijepalli, N.C., 2012. A method to assess demand growth vulnerability of travel times on road network links. Transport. Res. A 46 (5), 772-789.

Zhang, P., Peeta, S., 2011. A generalized modeling framework to analyze interdependencies among infrastructure systems. Transport. Res. B 45 (3), 553-579. 